\title{
Contrasting Effects of Spousal Education on Depressive Symptoms Among Korean Middle-Aged and Older Adults
}

\author{
Soong-Nang Jang ${ }^{1}$, Ichiro Kawachi ${ }^{2}$ \\ ${ }^{1}$ Red Cross College of Nursing, Chung-Ang University, Seoul, Korea, ${ }^{2}$ Department of Social Behavioral Sciences, Harvard T.H. CHAN School \\ of Public Health, Boston, MA, USA
}

Corresponding Author: Soong-Nang Jang, MPH, PhD Red Cross College of Nursing, Chung-Ang University, 84 Heukseok-ro, Dongjak-gu, Seoul 06974, Korea

Tel: +82-2-820-5806

Fax: +82-2-824-5967

E-mail: sjang@cau.ac.kr

Received: January 30, 2018

Revised: March 2, 2018

Accepted: March 10, 2018

\begin{abstract}
Background: Within marital dyads, we focused on the so-called positive spillover effects of one spousal partner's educational attainment on the health of the other partner. This study examined the relationship between spousal educational attainment and depressive symptoms in Korean older adults. Methods: Data were obtained from a sample of marital dyads aged 45 or older (total 6,824 husbands and wives) from the baseline survey of the Korean Longitudinal Study of Ageing. Depressive symptoms were measured by the 10-item Center for Epidemiological Studies Depression scale (CES-D 10). A stepwise actor-partner interdependence model was used to examine the association between own and spousal educational attainment and depressive symptoms, conditioning for covariates. Results: Among men, we found that their wives' educational attainment did not influence their mental health, whereas, among women, their depressive symptoms were inversely related to their husbands' level of schooling. With regard to own education, more-educated men reported lower psychological distress, whereas, among women, there was no overall association. However, in a subset of Korean women with the highest level of household income, higher educational attainment was associated with more depressive symptoms. Conclusion: Our findings underscore the need to incorporate the cultural context in examining the spillover effects of education on health within marriage.
\end{abstract}

Key Words: Depressive symptoms, Marital status, Educational level, Dyad analysis

\section{INTRODUCTION}

Marital status is a robust predictor of health status. From studies conducted in the Western context, 2 stylized facts have emerged: first, the married live longer than the unmarried (i.e., never married, widowed, separated, or divorced), and second, the health benefits of marriage appear to be greater for men compared to women. The health premium from marriage has, in turn, been ascribed to improved access to economic resources, household economies of scale, exchange of social support, and social influence stemming from the partner's health behaviors. ${ }^{1-3)}$

What remains less well understood is the heterogeneity in the influence of marriage on health according to socioeconomic status. Of particular interest is whether the benefits of marriage vary depending on the discrepancy between spouses' level of education. For example, is there a benefit from being married to a highly educated spouse even if one has a low level of educational attainment (the so-called positive "spillover" effect of education in marriage)? Some studies suggest that there is indeed evidence for such spillover effects. $^{4-7)}$

However, there is also evidence of a gender difference in the effects of spousal education on health. Thus, some United States (US) studies have suggested that men whose wives are more educated have an increased risk of coronary heart disease. ${ }^{8-12)}$ In the study by Suarez and Barrett-Connor, ${ }^{12)}$ being married to a more-educated wife was associated with increased risk of all-cause mortality among husbands. Using a nationally representative sample of the US population in 1992, Wilson ${ }^{13)}$ showed that the education level of the husband is positively associated with the self-assessed general health status of the wife while higher wife's education is associated with lower health status for her husband.

When we turn to social contexts and cultures outside of the West, the relationship between marriage and health becomes still more complex. Thus, while Western studies typically suggest that both men and women experience a health benefit from marriage (although the magnitude of the health benefit tends to be less for women), recent studies in Asia (Japan and Korea) have suggested that marriage may have no benefit for women or may even be detrimental (compared to the never married). ${ }^{14-16)}$ For example, in a 10-year 
prospective cohort study of 94,000 Japanese women and men aged 40-79, Ikeda et al. ${ }^{14)}$ reported that widowhood and divorce increased the risk of all-cause mortality among men 1.5-2.0 fold. By comparison, widowhood and divorce had no adverse effect on the mortality risk for Japanese women, suggesting that marriage was not protective for their physical health. In the Aichi Gerontological Evaluation Study cohort, Kondo ${ }^{17)}$ found a gradient in depressive symptoms that seems to be the opposite of what has been observed in the West. In that study, the highest levels of depressive symptoms were reported by currently married older women followed by never-married women, and the lowest levels were found among widowed/divorced women.

Although the reasons for the societal differences in the pattern of marriage and health remain obscure, one potential clue is provided by the cultural variations in the gender division of labor within the household, particularly the caregiver role. The strict gender division of roles in Asian culture also led us to postulate that there would be gender differences in the spillover effects of partner's level of education on an individual's health status. Korea has been slow to adapt to the new realities of women working outside the home. In this society, women are often expected to quit work when they marry; indeed, they are somewhat unique among highly industrialized countries in continuing to display the "M-shape" pattern of female labor force participation across the life course - i.e., women withdraw from the labor market during their childbearing and child-raising years. Being married to a more highly educated wife, therefore, does not necessarily translate to a higher household standard of living. Alternatively, the frustration of highly educated women may rub off on their husbands such that we predict that a wife's level of education would not lead to a positive spillover effect on a husband's level of mental health. Meanwhile, traditional Korean men continue to be assigned the "breadwinner" role and often have little involvement with caregiving roles or household labor. To the extent that higher levels of education among men predict more liberated attitudes and norms, we hypothesize that there is a positive spillover effect of husbands' educational attainment on their wives' mental health.

In the present study, we investigated the effects of spousal educational level on depressive symptoms among Korean marital dyads. We aimed to assess the separate and independent contributions of own level of education vs. spouse's level of education on depressive symptoms. We used the actor-partner interdependence model (APIM) ${ }^{18)}$ to take account of the clustering within marital dyads.

\section{MATERIALS AND METHODS}

\section{Data and Measures}

We used the 1st wave of the Korean Longitudinal Study of Ageing (KLoSA). The survey used a multistage stratified probability sample of Koreans aged 45 and older living in households. A total of 10,254 individuals completed interviews in 2006. The full information about the questionnaires, sampling methods, and weights can be obtained at KLoSA website (www.klosa.re.kr). We limited our analysis to 3,412 marital dyads who remained partnered since the 2006 baseline. Weights were assigned to the census units to estimate parameters, reflecting the sampling process, the survey process, and accuracy of external data.

The short-form (10-item) Center for Epidemiological Studies Depression scale (CES-D 10) served as the outcome variable. The CES-D 10 is a brief screening instrument that assesses depressive symptoms experienced during the most recent week. ${ }^{15,19,20)}$ The responses for each item ranged from 0 (very rarely or less than once a day) to 3 (almost always or 5-7 days during the past week). The sum of the 10 items served as the outcome measure. Higher scores indicated greater distress.

Own and spouse's educational levels were categorized as (1) elementary school or less, (2) middle school graduated, (3) high school graduated, and (4) college graduated and beyond. Household-equivalent income was calculated as the total household income divided by the square root of the number of household members; these scores were then divided into tertiles. Employment status was divided into 3 groups: (1) employed, (2) retired, and (0) not in the labor force (unemployed or homemaker). Living arrangement was categorized into (1) living with spouse only, (2) living with children or children's family, and (3) living with others. Individuals were asked to rate their health using the World Health Organization version of self-rated health $(1$, very good; 2 , good; 3 , fair; 4, bad; or 5 , very bad). Functional status was measured using the 7 items of Activities of Daily Living and 10 items of Instrumental Activities of Daily Living. We counted items answered as partially or fully dependent on others (ranged from 0 to 17). Cognitive function was assessed using the Mini-Mental Status Examination (MMSE).

\section{Statistical Analyses}

Age-adjusted least square means (LS means) of the CES-D 10 according to own and partner's educational levels were calculated by means of analysis of covariance. We also calculated LS Means of the CES-D 10 according to own and partner's educational level by household income level.

The APIM using multilevel linear models, a technique developed and applied for dyadic data analysis, ${ }^{21,22)}$ was then used to estimate the association between education and depressive symptoms. Data were analyzed in 4 stages: first examining the educational level of each spouse and then using three models including interaction terms. The first interaction term was introduced as a multiplicative term (husband's education 
$x$ wife's education). Second, we created a new term representing the absolute value of the difference between the partner's educational level, which is appropriate when the similarity between partners is of interest. ${ }^{18)}$ In the final model, the interactions between household income and own or partner's educational effects on depressive symptoms were included in the models. All models were adjusted for age, living arrangement, functional status, self-rated health, and MMSE score. All models were calculated using Proc Mixed separately by gender.

\section{RESULTS}

Women were younger than men in our marital dyads samples; $61.93 \%$ were $45-64$ years old among men, and $73.15 \%$ were 45-64 years old among women (Table 1). The educational distribution varied substantially between genders: $28.77 \%$ of men and $46.29 \%$ of women had educational attainment below elementary school level. As we expected, college or higher education is more frequent among men (19.57\%) than among women (6.58\%). Over half of the men $(57.90 \%)$ were employed, whereas $28.16 \%$ of the women were in the active labor force. Men were less likely to live with college-educated partners while women had more college-graduate husbands. Although men showed more functional limitation than did women, self-rated health, cognitive function, and depressive symptoms showed worse profiles among wives than among husbands.

We found a stepwise pattern in CES-D 10 LS means adjusting for age according to own educational level among men $(\beta=$ $-0.512, p<0.001)$, though not among women (Fig 1). When we turn to partner's education, men did not exhibit such a pattern, but women who were living with higher-educated husbands were less likely to report depressive symptoms $(\beta=$ $-0.529, p<0.001)$.

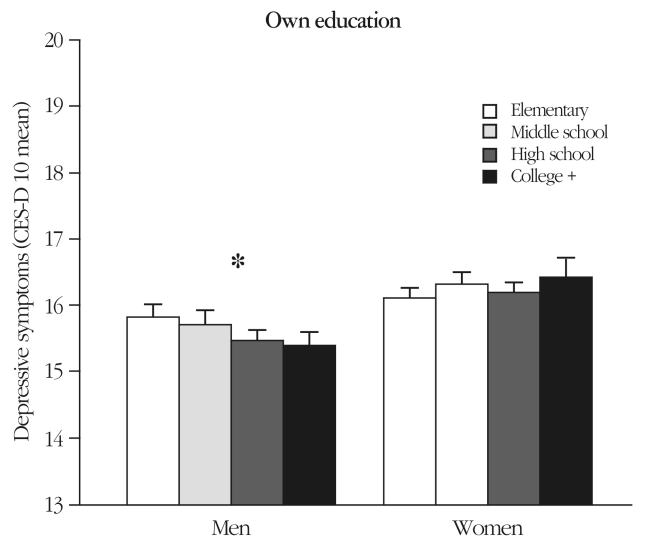

Table 1. General characteristics of marital dyads among Korean older adults in Korean Longitudinal Study of Ageing

\begin{tabular}{|c|c|c|}
\hline Characteristic & Men & Women \\
\hline \multicolumn{3}{|l|}{ Age (yr) } \\
\hline 45-64 & 61.93 & 73.15 \\
\hline$\geq 65$ & 38.07 & 26.85 \\
\hline \multicolumn{3}{|l|}{ Own educational level } \\
\hline Under elementary school & 28.77 & 46.29 \\
\hline Middle school graduated & 17.47 & 19.73 \\
\hline High school graduated & 34.18 & 27.41 \\
\hline College graduated or over & 19.57 & 6.58 \\
\hline \multicolumn{3}{|l|}{ Household income (tertiles)* } \\
\hline 1T (High) & 32.96 & 33.79 \\
\hline 2T (Middle) & 33.71 & 33.60 \\
\hline 3T (Low) & 33.32 & 32.61 \\
\hline \multicolumn{3}{|l|}{ Own job status } \\
\hline Employed & 57.90 & 28.16 \\
\hline Retired & 25.26 & 12.60 \\
\hline Unemployed & 16.84 & 59.24 \\
\hline \multicolumn{3}{|l|}{ Living arrangement } \\
\hline Couple & 43.52 & 43.52 \\
\hline Couple+children & 43.86 & 43.86 \\
\hline Couple+children+grandchildren & 11.10 & 11.10 \\
\hline Others & 1.52 & 1.52 \\
\hline Functional limitation & $0.763 \pm 2.654$ & $0.3857 \pm 1.945$ \\
\hline Self-rated health & $2.744 \pm 0.966$ & $2.955 \pm 0.959$ \\
\hline MMSE & $25.616 \pm 5.311$ & $24.901 \pm 5.382$ \\
\hline CES-D 10 & $15.677 \pm 4.410$ & $16.283 \pm 4.800$ \\
\hline
\end{tabular}

Values are presented as weighted $\%(n=3,412)$ or mean \pm standarde deviation.

MMSE, Mini-Mental Status Examination; CES-D 10, Short Form of Center for Epidemiological Survey of Depression 10 items. *Tertiles of household equivalent income.

Fig. 1. Depressive symptoms by own and partner's educational levels among Korean men and women. Least-squares means of CES-D 10 scores adjusting for age, $" \mathrm{p}<0.05$ by analysis of covariance. CES-D 10, Short Form of Center for Epidemiological Survey of Depression 10 items. 
Table 2 presents the associations of own and partner's educational level with depressive symptoms. Among men, own educational level was inversely associated with depressive symptoms. Men with lower levels of schooling were more likely to report depressive symptoms; however, there was no association of wife's educational level with husband's depression (model 1 in Table 2). By contrast, women showed no association between own educational level and depressive symptoms, which was different from the pattern found in men. In additional analyses, we examined the interaction term between own and partner's educational levels, but there was no significant association within marital dyads. Similarity of educational level calculated by the absolute value of the educational disparity difference between spouses also showed no association with depression in either husbands or wives (models 3 and 4).

Household income was strongly associated with depressive symptoms among both men and women (model 2). Model 5 presents the results of the interaction between household income, own educational level, and partner's educational level. Only among women, household income and own educational level showed a significant interaction $(\beta=0.455, p<0.01)$; no significant interaction was shown between husband's education and household income. In additional analysis, we stratified models by age group - middle-aged people 45-64 years old versus those aged 65 years or older - to look for any differences between age groups. We found that middle-aged women showed similar results as the total sample, but not older women. In other words, linkage to husband's educational level appeared to be stronger among middle-aged

Table 2. Stepwise actor-partner interdependence model models for the association of actor and partner's educational level with depressive symptoms among Korean men and women

\begin{tabular}{|c|c|c|c|c|c|}
\hline Variable & Model 1 & Model 2 & Model 3 & Model 4 & Model 5 \\
\hline \multicolumn{6}{|l|}{ Men } \\
\hline Own education & $-0.512(0.094)^{* * * *}$ & $-0.268(0.087)^{* * *}$ & $-0.220(0.154)$ & $-0.100(0.143)$ & $-0.397(0.130)^{* * *}$ \\
\hline Partner's education & $0.102(0.114)$ & $0.203(0.104)$ & $0.300(0.275)$ & $0.042(0.150)$ & $0.212(0.161)$ \\
\hline Interaction of education $(\mathrm{O} \times \mathrm{P} \text { education })^{\dagger}$ & - & - & $-0.031(0.081)$ & - & - \\
\hline Similarity of education $(\mid \mathrm{O}-\mathrm{P} \text { education } \mid)^{\dagger}$ & - & - & - & $-0.226(0.153)$ & - \\
\hline Household income tertiles & - & $-0.313(0.090)^{* * * *}$ & $-0.311(0.090)^{* * *}$ & $-0.314(0.090)^{* * *}$ & $-0.627(0.220)^{* * *}$ \\
\hline Own education $\times$ income & - & - & - & - & $0.149(0.108)$ \\
\hline Partner's education $\times$ income & - & - & - & - & $-0.032(0.118)$ \\
\hline Age & $0.091(0.009)^{* * * *}$ & $-0.014(0.009)$ & $-0.014(0.009)$ & $-0.014(0.009)$ & $-0.015(0.009)$ \\
\hline Own job status & - & $0.694(0.102)^{* * * *}$ & $0.695(0.102)^{* * * * *}$ & $0.698(0.102)^{* * * *}$ & $0.694(0.102)^{* * * *}$ \\
\hline Partner's job status & - & $0.031(0.078)$ & $0.031(0.078)$ & $0.032(0.078)$ & $0.028(0.078)$ \\
\hline Physical functions & - & $0.302(0.029)^{* * * *}$ & $0.301(0.029)^{* * * *}$ & $0.301(0.029)^{* * * *}$ & $0.303(0.029)^{* * * *}$ \\
\hline Self-rated health & - & $1.327(0.083)^{* * * *}$ & $1.328(0.083)^{* * * * *}$ & $1.324(0.083)^{* * * *}$ & $1.321(0.083)^{* * * *}$ \\
\hline MMSE & - & $-0.088(0.015)^{* * * *}$ & $-0.089(0.015)^{* * * *}$ & $-0.088(0.015)^{* * * *}$ & $-0.088(0.015)^{* * * *}$ \\
\hline Social participation & - & $-0.471(0.191)^{*}$ & $-0.471(0.191)^{*}$ & $-0.470(0.191)^{*}$ & $-0.468(0.191)^{*}$ \\
\hline Living arrangement & - & $0.012(0.093)$ & $0.012(0.093)$ & $0.010(0.093)$ & $0.018(0.094)$ \\
\hline \multicolumn{6}{|l|}{ Women } \\
\hline Own education & $-0.111(0.121)$ & $0.226(0.112)^{*}$ & $0.665(0.295)^{*}$ & $0.426(0.162)^{* * *}$ & $-0.269(0.174)$ \\
\hline Partner's education & $-0.529(0.100)^{* * * *}$ & $-0.224(0.093)^{*}$ & $-0.007(0.164)$ & $-0.433(0.154)^{* * *}$ & $-0.027(0.139)$ \\
\hline Interaction of education $(\mathrm{O} \times \mathrm{P} \text { education })^{\dagger}$ & - & - & $-0.139(0.086)$ & - & - \\
\hline Similarity of education $(\mid \mathrm{O}-\mathrm{P} \text { education } \mid)^{\dagger}$ & - & - & - & $0.280(0.164)$ & - \\
\hline Household income tertiles & - & $-0.559(0.095)^{* * * *}$ & $-0.553(0.095)^{* * * *}$ & $-0.553(0.095)^{* * * *}$ & $-0.967(0.234)^{* * * *}$ \\
\hline Own education $\times$ income & - & - & - & - & $0.455(0.126)^{* * *}$ \\
\hline Partner's education $\times$ income & - & - & - & - & $-0.204(0.115)$ \\
\hline Age & $0.096(0.010)^{* * * *}$ & $-0.018(0.010)$ & $-0.016(0.010)$ & $-0.018(0.010)$ & $-0.020(0.010)$ \\
\hline Own job status & - & $0.148(0.083)$ & $0.146(0.083)$ & $0.146(0.083)$ & $0.157(0.083)$ \\
\hline Partner's job status & - & $0.292(0.106)^{* * *}$ & $0.294(0.106)^{* * *}$ & $0.287(0.106)^{* * *}$ & $0.300(0.106)^{* * *}$ \\
\hline Physical functions & - & $0.313(0.044)^{* * * *}$ & $0.312(0.044)^{* * * *}$ & $0.314(0.044)^{* * * *}$ & $0.309(0.044)^{* * * *}$ \\
\hline Self-rated health & - & $1.647(0.086)^{* * * *}$ & $1.645(0.086)^{* * * *}$ & $1.644(0.086)^{* * * *}$ & $1.647(0.086)^{* * * *}$ \\
\hline MMSE & - & $-0.122(0.016)^{* * * *}$ & $-0.124(0.016)^{* * * *}$ & $-0.123(0.016)^{* * * *}$ & $-0.120(0.016)^{* * * *}$ \\
\hline Social participation & - & $-0.201(0.170)$ & $-0.184(0.171)$ & $-0.194(0.170)$ & $-0.205(0.170)$ \\
\hline Living arrangement & - & $0.182(0.099)$ & $0.180(0.099)$ & $0.184(0.099)$ & $0.201(0.099)^{*}$ \\
\hline
\end{tabular}

Values are presented as parameter estimate (standard error).

Model 1 included age. Model 2 adjusted for age, functional status, self-rated health, Mini-Mental Status Examination (MMSE), living arrangement, employment status. Model 3 included interaction of education and model 2 covariates. Model 4 included similarity of education and model 2 covariates. model 5 adjusted model 2 covariates and interaction of educational level and household income.

* $\mathrm{p}<0.05$. * $\mathrm{p}<0.01$. ${ }^{* * \mathrm{p}}<0.001$. Interaction of education $=$ own education $\times$ partner's education. ${ }^{\dagger}$ Similarity of education $=$ own educationpartner's education. 

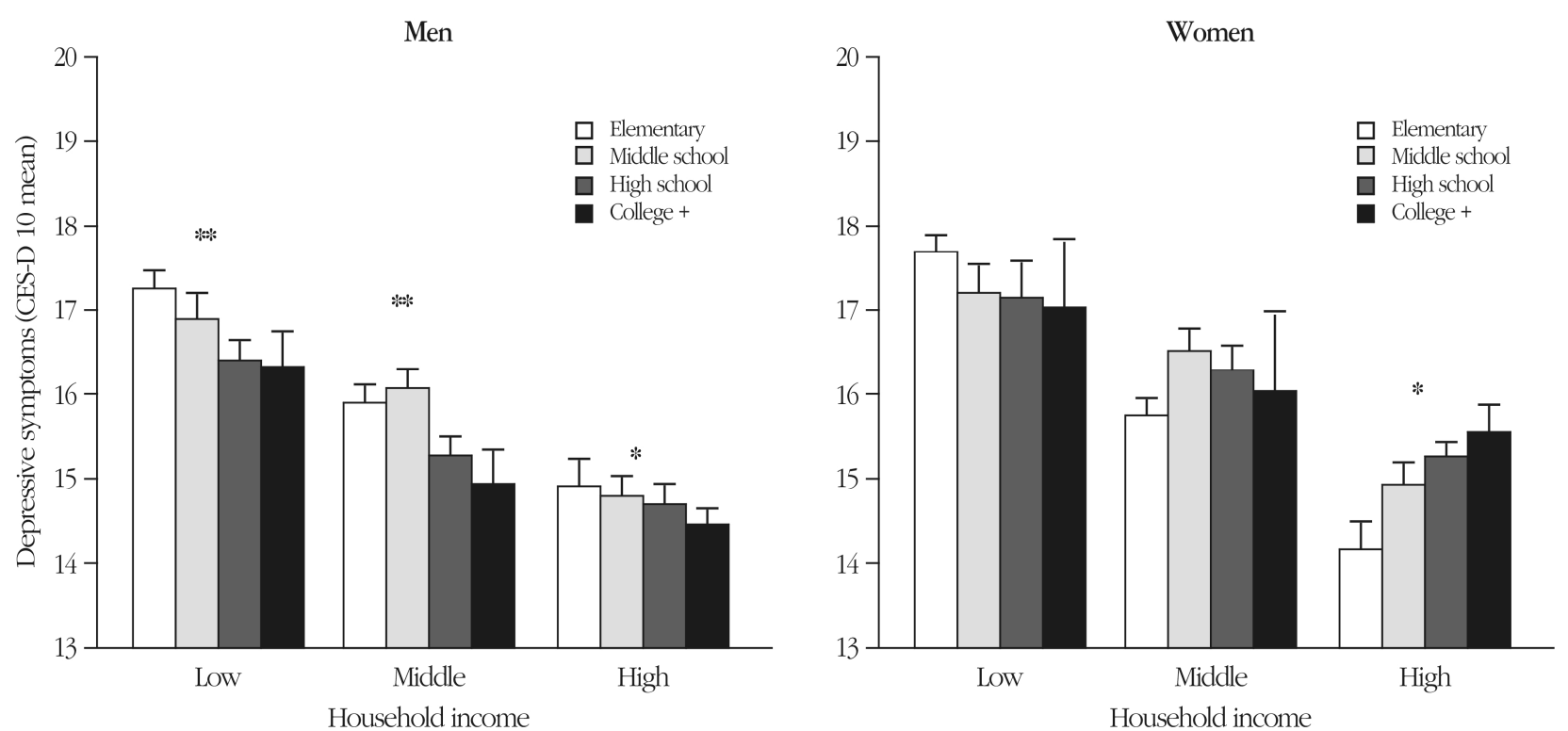

Fig. 2. Depressive symptoms scores (least square means of CES-D 10) by own educational level in each household income level (low, medium, and high) among Korean men and women. CES-D 10, Short Form of Center for Epidemiological Survey of Depression 10 items.

$* \mathrm{p}<0.05, * \mathrm{p}<0.01$ by analysis of covariance.

women than among older women. Since the directions of coefficients were similar in both age groups, we combine age groups in Table 2 .

Fig. 2 shows the gradient in CES-D 10 LS means adjusting for age by educational level according to household income level among men and women. In contrast to the clear gradient in men's depressive symptoms (lower education=higher symptoms scores) at each household income level, women's depression showed a different pattern. We could not detect any pattern among women in low or medium household income strata. Interestingly, however, women who were in the highest household income strata were more likely to report depressive symptoms as their educational level increased $(\beta=0.93, p=0.03)$.

\section{DISCUSSION}

The results of our analyses reveal four sets of patterns associated with depressive symptoms within marital dyads in Korea. First, own level of education was inversely associated with depression among men but not women. Second, partner's level of education was inversely associated with depression among women but not men. Third, we did not find any association between within-dyad educational discrepancy and depressive symptoms in either men or women. Fourthly, when we stratified couples by household level of income, educational attainment was consistently inversely associated with depressive symptoms among men. However, among women in the highest stratum of household income, more schooling was associated with higher depressive symp- toms.

Two alternative explanations are possible for the positive spillover effects of husband's education level on wives' mental health. First, since the husband's educational level more strongly determines the household's socioeconomic circumstances, the level of schooling of the male head of household may be a marker for household income. ${ }^{23)}$ However, even after we adjusted for household income, husband's education continued to be associated with lower depressive symptoms among wives, and, in fact, the coefficient of the estimate was slightly changed. This suggests that something other than economic circumstances explains the positive spillover of husband's education on wives' mental health. The alternative explanation is that more-educated husbands are more "liberated" in their attitudes towards the traditional division of labor in the household in Korea. ${ }^{24)}$ Although we did not measure the gendered division of household labor (or attitudes toward the same), future studies are warranted to test this hypothesis.

By contrast, we could not detect any influence of wives' educational level on husbands' mental health. This pattern is in marked contrast to recent studies from the West, which suggested that the wife's level of educational attainment is an even stronger predictor of her husband's risk of dying than his own level of schooling. ${ }^{4-7)}$ Jaffe et al., ${ }^{5)}$ in a study based in Israel, demonstrated that a wife's educational attainment appeared to be a stronger predictor of her husbands' cardiovascular disease mortality then his own educational level while, for women, a husband's education had little 
or no effect.

With regard to the influence of own educational attainment, we found that Korean women's levels of depressive symptoms are not linked to their levels of schooling. This may be partly accounted for by the heterogeneous pattern revealed in Fig 2, viz., the education/mental health gradient runs in opposite directions for wives depending on their level of household income. Notably, among women in the highest income stratum, more-educated women report worse levels of depressive symptoms. This may reflect the high levels of frustration and truncated career aspirations among Korean women in this social class. Interestingly, recent Japanese data reveal a similar pattern of worse health status among highly educated women. Working women with higher educational levels have a higher level of perceived psychological stress, which translates to an increased risk of stroke among working highly educated women. ${ }^{25)}$

Finally, although studies conducted in Western contexts have reported that husbands married to wives with higher levels of education may experience adverse health outcomes, ${ }^{12,26)}$ we did not find such a pattern in Korea. However, this may simply reflect the rarity of this type of arrangement. Because of the lower levels of educational attainment among Korean women from older generations, too few couples exhibited this pattern of educational discrepancy to provide an adequate statistical test of this hypothesis. As Korean women attain higher levels of schooling, future studies will be able to test this idea in Korean society.

Depression is a common mental health problem among older adults. About 12\%-35\% of older persons experience depressive symptoms requiring intervention in Korea, rates that were higher than those in Western countries. ${ }^{27)}$ In Korea, the suicide rate among elders has been increasing at an alarming rate since 1990, and the country ranked at the top of OECD countries in recent years. Based on our results, less-educated men, women married to less-educated men, and elders in low-income households are at increased risk of depression. Thus, understanding psychological distress according to socioeconomic circumstances and familial roles within marital dyads is necessary to reduce depression between husbands and wives and to enable both members of an older couple to receive equal mental health benefits from marriage.

Several limitations warrant consideration in generalizing our observation. In our data, we lacked information on the length of the union (when it started or was dissolved) among couples. It seems likely that the longer the marriage, the larger the influence of a partner's socioeconomic position on the spouse's depression status. Because this was an observational study, the researchers could not rule out endogeneity and selection; i.e., individuals who were already depressed may have elected to live with low-educated partners.
On the other hand, it is harder to attribute the observed, stark gender difference in risk to selection and endogeneity alone.

In conclusion, among marital dyads, the educational level of the partners exhibited a gendered pattern of association with depressive symptoms. We found that a less-educated husband increases the wife's depression risk as well as his own depression risk. Although the husband may determine the economic level of the household, there may be additional considerations besides economic circumstances for a potential positive spillover effect of husband's education, such as more liberated attitudes towards the traditional division of labor in the household.

Conflicts of Interest Disclosures: The researchers claim no conflicts of interest.

\section{REFERENCES}

1. Eng PM, Kawachi I, Fitzmaurice G, Rimm EB. Effects of marital transitions on changes in dietary and other health behaviours in US male health professionals. J Epidemiol Community Health 2005;59:56-62.

2. House JS, Landis KR, Umberson D. Social relationships and health. Science 1988;241:540-5.

3. Waite LJ. Does marriage matter? Demography 1995;32:483-507.

4. Bosma H, Appels A, Sturmans F, Grabauskas V, Gostautas A. Educational level of spouses and risk of mortality: the WHO Kaunas-Rotterdam Intervention Study (KRIS). Int J Epidemiol 1995;24:119-26.

5. Jaffe DH, Eisenbach Z, Neumark YD, Manor O. Does one's own and one's spouse's education affect overall and cause-specific mortality in the elderly? Int J Epidemiol 2005;34:1409-16.

6. Jaffe DH, Eisenbach Z, Neumark YD, Manor O. Effects of husbands' and wives' education on each other's mortality. Soc Sci Med 2006;62:2014-23.

7. Skalická V, Kunst AE. Effects of spouses' socioeconomic characteristics on mortality among men and women in a Norwegian longitudinal study. Soc Sci Med 2008;66:2035-47.

8. Bruhn JG, Chandler B, Lynn TN, Wolf S. Social characteristics of patients with coronary heart disease. Am J Med Sci 1966; 251:629-37.

9. Shekelle RB. Educational status and risk of coronary heart disease. Science 1969;163:97-8.

10. Medalie JH, Snyder M, Groen JJ, Neufeld HN, Goldbourt U, Riss E. Angina pectoris among 10,000 men. 5 year incidence and univariate analysis. Am J Med 1973;55:583-94.

11. Haynes SG, Eaker ED, Feinleib M. Spouse behavior and coronary heart disease in men: prospective results from the Framingham heart study. I. Concordance of risk factors and the relationship of psychosocial status to coronary incidence. Am J Epidemiol 1983;118:1-22.

12. Suarez L, Barrett-Connor E. Is an educated wife hazardous to your health? Am J Epidemiol 1984;119:244-9.

13. Wilson SE. The health capital of families: an investigation of 
the inter-spousal correlation in health status. Soc Sci Med 2002; 55:1157-72.

14. Ikeda A, Iso H, Toyoshima H, Fujino Y, Mizoue T, Yoshimura $\mathrm{T}$, et al. Marital status and mortality among Japanese men and women: the Japan Collaborative Cohort Study. BMC Public Health 2007;7:73.

15. Jang SN, Kawachi I, Chang J, Boo K, Shin HG, Lee H, et al. Marital status, gender, and depression: analysis of the baseline survey of the Korean Longitudinal Study of Ageing (KLOSA). Soc Sci Med 2009;69:1608-15.

16. Jeon GS, Jang SN, Rhee SJ, Kawachi I, Cho SI. Gender differences in correlates of mental health among elderly Koreans. J Gerontol B Psychol Sci Soc Sci 2007;62:S323-9.

17. Kondo K. Exploring "Inequalities in Health": a large-scale social epidemiological survey for care prevention in Japan. Tokyo: Igaku-Shoin Ltd.; 2007. p. 22.

18. Campbell L, Kashy DA. Estimating actor, partner, and interaction effects for dyadic data using PROC MIXED and HLM: a user-friendly guide. Person Relatsh 2002;9:327-42.

19. Irwin M, Artin KH, Oxman MN. Screening for depression in the older adult: criterion validity of the 10-item Center for Epidemiological Studies Depression Scale (CES-D). Arch Intern Med 1999;159:1701-4.

20. Kohout FJ, Berkman LF, Evans DA, Cornoni-Huntley J. Two shorter forms of the CES-D (Center for Epidemiological Studies
Depression) depression symptoms index. J Aging Health 1993; 5:179-93.

21. Kenny DA, Kashy DA. The design and analysis of data from dyads and groups. In: Reis HT, Judd CM, editors. New York: Cambridge University Press; 2000. p.589-607.

22. Kenny DA, Cook W. Partner effects in relationship research: conceptual issues, analytic difficulties, and illustrations. Person Relatsh 1999;6:433-48.

23. Monden CW, van Lenthe F, de Graaf ND, Kraaykamp G. Partner's and own education: does who you live with matter for self-assessed health, smoking and excessive alcohol consumption? Soc Sci Med 2003;57:1901-12.

24. Kim TH, Jun GY. A study on the conjugal support, the share of household activities and marital adjustment in old age. J Korean Gerontol Soc 1997; 17:167-82.

25. Honjo K, Iso H, Inoue M, Tsugane S; Japan Public Health Centerbased Prospective Study Group. Education, social roles, and the risk of cardiovascular disease among middle-aged Japanese women: the JPHC Study Cohort I. Stroke 2008;39:2886-90.

26. Egeland GM, Tverdal A, Meyer HE, Selmer R. A man's heart and a wife's education: a 12-year coronary heart disease mortality follow-up in Norwegian men. Int J Epidemiol 2002;31:799805.

27. Park JH, Kim KW. A review of the epidemiology of depression in Korea. J Korean Med Assoc 2011;54:362-9. 